\title{
Socioeconomic status is an independent predictor of biochemical recurrence among patients with prostate cancer who undergo radical prostatectomy
}

\author{
Victor Srougi, Alberto A. Antunes, Sabrina T, Reis, Marcos F. Dall'Oglio, Adriano J. Nesrallah, \\ Kátia R. M. Leite, Miguel Srougi
}

Division of Urology, University of Sao Paulo Medical School, Sao Paulo, Brazil

\begin{abstract}
Purpose: Socioeconomic status (SES) may influence cancer characteristics and behavior in several aspects. We analyzed PCa characteristics and behavior among low income uninsured men, and compare them to high income patients with health insurance in a developing country.

Materials and Methods: A retrospective case-control study was performed on 934 patients with clinically localized PCa who underwent radical prostatectomy between March, 1999 and July, 2009. Patients were divided in two groups, according to their SES. In group $1(\mathrm{n}=380)$, all had low income, low educational levels and couldn't afford medical insurance. In group $2(\mathrm{n}=554)$, all had higher income, higher education and had medical insurance.

Results: Patients from group 1 were older, had higher Gleason scores, higher rates of seminal vesicle and bladder neck involvement. The Kaplan Meier disease-free survival curve demonstrated that after a follow-up of four years, about $50 \%$ of uninsured patients had biochemical recurrence, versus $21 \%$ of insured patients (Log rank test: $p<0.001$ ). A multivariate Cox regression analysis for the risk of disease recurrence demonstrated that only PSA levels, Gleason score, seminal vesicle involvement and SES were statistically significant variables. Patients with a low SES presented 1.8 times the risk of recurrence as compared to patients with a high SES.

Conclusions: Patients with low SES were older, presented more aggressive PCa characteristics and a high rate of disease recurrence. A low SES constituted an independent predictor for disease recurrence.
\end{abstract}

Key words: prostate-specific antigen; prostate; neoplasms; socioeconomic factors; prostatectomy; survival

Int Braz J Urol. 2011; 37: 507-513

\section{INTRODUCTION}

Prostate cancer $(\mathrm{PCa})$ remains the most common non-cutaneous tumor in incidence and the second in mortality among North American men. It is estimated 192,280 new cases and 27,360 deaths in USA in 2009 (1). Being 13\% of all cancers that affect men, it has a great socioeconomic importance. The widespread use of prostatic specific antigen (PSA) in PCa screening during the last two decades has led to an increase in diagnosis of localized disease, with mortality declining in synchrony with this risk migration (2).

Socioeconomic status (SES) may influence cancer characteristics and behavior in several aspects, mainly due to its influences on lifestyle and exposure to environmental risk factors, as well as access and utilization of health care services (35). Clegg et al. (5), analyzing 15,357 patients from the Surveillance, Epidemiology, and End Results (SEER) database and the National Longitudinal Mortality Study (NLMS) reported greater rates of 
lung cancer and an increased incidence of distantstage breast and $\mathrm{PCa}$ among low-income patients. Further data regarding PCa have reported higher mortality rates among patients in the low levels of SES (4). Likewise, recent studies have demonstrated that despite the widespread adoption of PSA screening, the diagnosis of low-risk PCa has not been increasing among men in lower socioeconomic status (6).

However, all studies to date regarding this issue were conducted in developed countries like United States of America. The influence of SES in $\mathrm{PCa}$ characteristics and behavior in a developing country with high socioeconomic disparities and high levels of poverty had never been studied so far. Finding a different behavior in the extremes of socioeconomic layers could help to improve PCa screening and treatment among these patients.

The aim of the present study is to analyze PCa characteristics and behavior among low-income uninsured men and compare these data to high-income patients with health insurance, in a developing country.

\section{MATERIALS AND METHODS}

A retrospective case-control analysis was performed on 934 patients with clinically localized PCa who underwent radical prostatectomy between March, 1999 and July, 2009. Patients were divided in two groups, according to their SES. In group 1 (study group) $(\mathrm{n}=380)$, all had low-income, low educational levels and couldn't afford medical insurance. These patients were treated in the Brazilian public health system (SUS). SUS is supposed to provide free and comprehensive PCa treatment for Brazilians without medical insurance, but due to high demand the service offered is suboptimal. In group 2 (control group) $(n=554)$, all had high-income, higher education and medical insurance. The latter were treated in private hospitals. Radical prostatectomy was performed by the same surgeons in both groups. Patients who had received any previous treatment for $\mathrm{PCa}$ were not considered for analysis. To avoid any selection bias, patients from both groups were offered the option of undergoing radiotherapy. Adjuvant radiation therapy was indicated to those cases with a gross positive surgical margin.

Data analyzed included patient age, serum total PSA, pathological stage, tumor grade, seminal vesicle involvement, urethral margin and bladder neck margin status. The 2002 TNM classification was applied for pathological staging (7). Preoperative clinical staging was performed through digital rectal examination, pelvic computerized tomography $(\mathrm{CT})$ and bone scintighraphy in most patients. Patients from both groups considered to have a lowrisk disease (PSA $<10 \mathrm{ng} / \mathrm{mL}$ and Glason score $<7$ and a T1c disease) did not undergo imaging studies. For analysis of pathology grade, we used the Gleason score (8). All the characteristics were compared in order to determine differences between the two groups. Staff pathologists with expertise in genitourinary pathology examined all specimens and margins, according to previous published protocols. Obturatory lymphadenectomy was performed only in intermediate and high-risk patients.

For statistical analysis we used the program SPSS version 15.0. Comparison of variables between the two groups, were performed through the Pearson Chi-square and Fisher's exact tests. For analysis of disease free survival, we constructed a Kaplan-Meier actuarial curve. Biochemical recurrence was defined as a PSA of $0.4 \mathrm{ng} / \mathrm{mL}$ or greater after radical prostatectomy. To determine the independent variables related to the risk of disease recurrence, we used a Cox regression model. For statistical analysis we considered a 5\% significance level to reject the hypothesis of a casual correlation $(\mathrm{p}<0.05)$.

\section{RESULTS}

At the moment of surgery, while $61.8 \%$ of patients from group 1 were 65 years old or older, the majority or $53.3 \%$ of patients from group 2 were younger than 65 years old $(p<0.001)$. Median age was 64 yo (40 to 83) for patients from the high SES group and 67 yo (44 to 81) for patients from the low SES group. Among patients from group 1, 41.9\% had a Gleason score of 7 or greater, while $37.9 \%$ of patients from group 2 had Gleason scores higher than $7(p=0.001)$. Seminal vesicle involvement was found in $11.6 \%$ and 
$6.3 \%$ of patients from group 1 and 2 respectively $(\mathrm{p}=$ 0.004). We didn't find significant difference regarding PSA levels and pathological stage between the two groups, with the majority of patients from both groups presenting PSA $<10.0 \mathrm{ng} / \mathrm{mL}$ and organ-confined disease (T2). Positive urethral margins were more common among patients from group 2 and positive bladder neck margins were more common among patients from group 1. In the first case, we believe this fact was rather due to efforts to spare external sphincter than to an advanced disease status. Thus, in most cases they were considered false positive margins. Characteristics of the two groups are described in Table-1.

The Kaplan Meier disease-free survival curve demonstrated that at four years, while $50 \%$ of patients from group 1 had biochemical recurrence, versus $21 \%$ of patients from group $2(p<0.001)$ (Figure-1). A multivariate Cox regression analysis for the risk of disease recurrence considering all the analyzed variables demonstrated that only serum PSA levels, Gleason score, seminal vesicle status and SES were independent variables. Patients in a low SES presented 1.8 times higher risk of recurrence as compared to patients belonging to high SES (Table-2).

\section{DISCUSSION}

In the present study, we demonstrated that a low SES is associated with more aggressive pathological characteristics of PCa. Patients in the low SES group were older, presented higher Gleason scores and higher rates of seminal vesicle and bladder neck involvement. Biochemical recurrence rates were higher among low SES group. In the Cox regression model for the risk of recurrence considering all the analyzed variables, only PSA levels,

Table 1 - Clinical and pathological characteristics of patients.

\begin{tabular}{|c|c|c|c|}
\hline & $\begin{array}{c}\text { Group } 1 \\
\text { (low S ES) } 380 \text { (\%) }\end{array}$ & $\begin{array}{c}\text { Group 2 } \\
\text { (high S ES) } 554 \text { (\%) }\end{array}$ & $\mathbf{p}$ \\
\hline Patient age (y ears) & & & $<0.001$ \\
\hline$<65$ & $145(38.2 \%)$ & $290(52.3 \%)$ & \\
\hline$\geq 65$ & $235(61.8 \%)$ & $264(47.7 \%)$ & \\
\hline PSA (ng/mL) & & & 0.599 \\
\hline$<10$ & $244(64.4 \%)$ & $342(61.7 \%)$ & \\
\hline $10-20$ & $100(26.4 \%)$ & $163(29.4 \%)$ & \\
\hline$>20$ & $35(9.2 \%)$ & $49(8.8 \%)$ & \\
\hline Pathological stage & & & 0.285 \\
\hline $\mathrm{T} 2$ & $272(71.8 \%)$ & $415(74.9 \%)$ & \\
\hline T3 & $107(28.2 \%)$ & $139(25.1 \%)$ & \\
\hline Gleason score & & & 0.001 \\
\hline$<7$ & $193(50.9 \%)$ & $344(62.1 \%)$ & \\
\hline$\geq 7$ & $186(49.1 \%)$ & $210(37.9 \%)$ & \\
\hline Seminal vesicle involvement & $44(11.6 \%)$ & $35(6.3 \%)$ & 0.004 \\
\hline Positive urethral margin & $52(13.7 \%)$ & $113(20.4 \%)$ & 0.009 \\
\hline Positive bladder neck margin & $22(5.8 \%)$ & $8(1.4 \%)$ & $<0.001$ \\
\hline
\end{tabular}




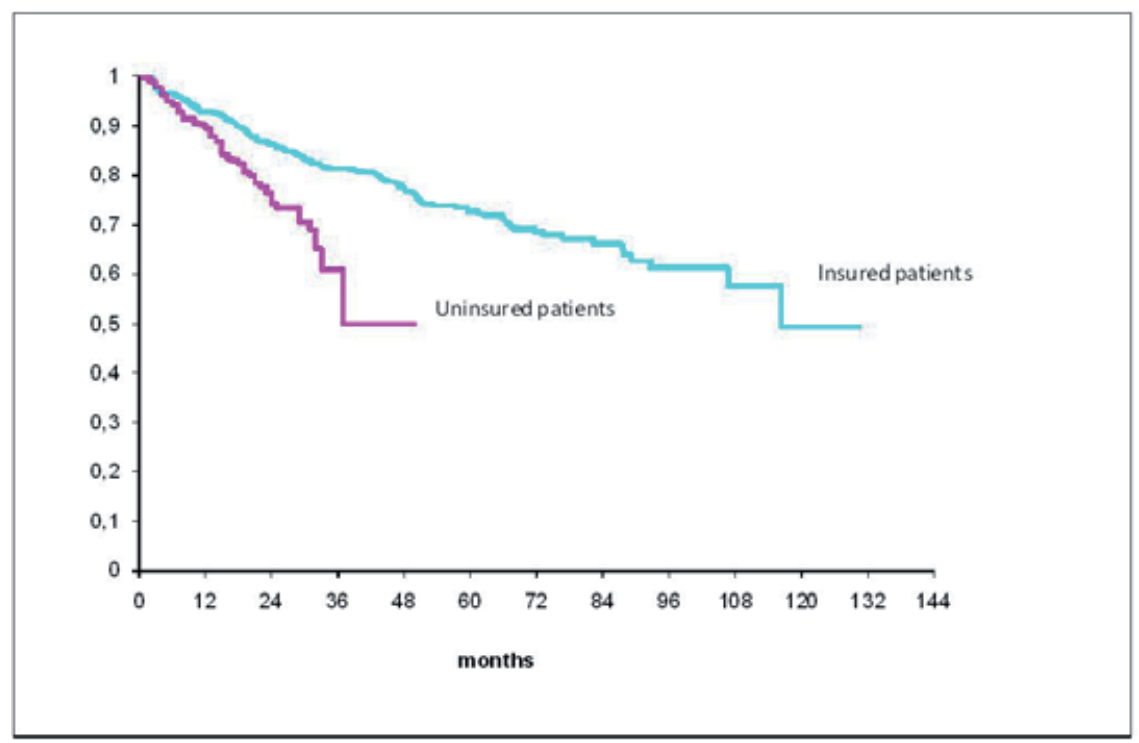

Figure 1 - Kaplan-Meier disease free survival curve.

Gleason score, seminal vesicle involvement and the SES were independent predictors. A low SES was associated with a risk of biochemical recurrence 1.8 times greater when compared to patients from the high SES group.

There are several studies correlating SES with cancer incidence and deaths, including all sites. In accordance to our results, Miller et al. (6), performed a retrospective cohort study of 570 disadvantaged men with diagnosis of PCa in California. They showed that $51 \%$ of the enrollees had a PSA greater than $10 \mathrm{ng} / \mathrm{mL}, 50 \%$ had a Gleason score of 7 or greater and $43 \%$ had a T2 or greater clinical stage. Metastasis at the diagnosis was present in $19 \%$ of the patients. The proportion of men with low-risk tumors was $24 \%$ and interestingly, this rate didn't increase over time. A limitation of this study was the lack of a control group. Cheng et al. (4), showed that a high SES is associated with higher incidence, but lower PCa mortality rate. It is known that SES is linked with several environmental dependent factors, including education, health care access and information. All together these factors may lead to lack in early detection and delay between PCa diagnosis and treatment among low SES patients.

The present study was conducted in Brazil, a country where $\mathrm{PCa}$ is also the highest prevalent cancer with socioeconomic disparities (9). The Bra- zilian health care system has two categories: private and public system. High-income population mainly uses the private system, which provides adequate screening and treatment against $\mathrm{PCa}$ for most patients. PSA screening is not formally performed in Brazil, but most patients from higher SES group choose to have it annually. This fact is not usually observed among patients with a low SES, which may lead to a delay in diagnosis. Lower SES patients use the public health care system, which due to higher demand, lacks in continuous screening, early diagnosis and may have delay in treatment. Despite these characteristics, in the present series serum PSA levels and pathological stages were similar between the groups. Additionally, it's important to note that in the present series median time to treatment was only slightly longer for patients from the low SES group (three months) as compared to the high SES group (1.5 months). Considering a tumor with such a long time to progression, we don't believe this small difference could be the reason to explain such a worse outcome among patients from the low SES group. It's possible that a longer time to diagnosis among these patients have influenced outcomes. However, since we received patients with confirmed $\mathrm{PCa}$ diagnosis we could not estimate the time.

Additionally, the low SES group is less exposed to health care information and is unlikely to 
Table 2 - Postoperative Result Assessment Criteria.

\begin{tabular}{llcc}
\hline Variable & HR & 95\% CI & p \\
\hline SES & 1.8 & 1.3 to 2.4 & $<0.001$ \\
PSA $10-20$ x PSA $<10$ & 1.5 & 1.1 to 2.0 & 0.004 \\
PSA $>20$ xSA $<10$ & 2.4 & 1.7 to 3.6 & $<0.001$ \\
Gleason score & 1.3 & 1.2 to 1.5 & $<0.001$ \\
Seminal vesicle involvement & 2.9 & 2.0 to 4.1 & $<0.001$ \\
\hline
\end{tabular}

undergo screening tests. This might be the reason why patients from the high SES group are usually diagnosed at an earlier age as we found in this series. All factors may lead to a more advanced disease at time of diagnosis, even among the clinically localized disease group.

It's important to note that mean follow-up was not equivalent between the groups. Despite longer follow up, the high SES group had better diseasefree survival than the low SES group. Explanations for these observations are uncertain but further studies controlling these variables should be made in order to conclude if the worst prognosis among patients from low SES group is due to health care deficiency or due to other factor. Another valuable study would be comparison of PCa stage at the moment of diagnosis, among extremes of SES, with the intention to analyze the disparities of the screening tests application between these groups. A possibility that could explain our results is that environmental differences could explain the more advanced Gleason scores and greater rates of seminal vesicle involvement among patients from the low SES group.

Margin status was significantly different between the two groups. While positive urethral margins were more common among patients from group 2 , positive bladder neck margins were more common among patients from group 1. It's well described that margin status is an independent predictor of PSA recurrence and secondary cancer treatment in patients who underwent radical prostatectomy (10). However, the location of positive surgical margins after surgery may significantly affect disease progression. Obek et al. (11), showed that a positive margin involving the bladder neck (which was more common among patients from low SES group) of the specimen carried a higher risk of progression. Despite these data, on multivariate analysis, margin status wasn't a significant predictor of disease recurrence.

Mean PSA levels were statistically similar between the two groups as well as pathological stage. Previously Kato et al. (12) related that PSA level is proportional to prostate tumor volume, and since all cases selected had localized disease, PSA tend to be in the same range in both groups. Staging was a pre-selection variable, justifying the similarities between the two groups.

Some limitations of the present study must be commented. We didn't have available data regarding the racial/ethnic distribution of both groups. It's well known that there is a higher PCa incidence and mortality among African Americans $(13,14)$. However, it remains poorly defined the reasons to explain this phenomenon and to what extent racial/ ethnic differences in PCa result from differences in SES. Tewari et al. (15), showed that socioeconomic factors are sufficient to explain the disparity in survival between white and black patients. While in univariate analysis, black patients had a significantly increased cancer-specific and overall mortality, after adjusting for insurance status and income on multivariate analysis, the two groups revealed no significant differences. It's also important to emphasize, that Brazil has a great variety of races and for this reason we believe that this variable could not have influenced the results. Another limitation is that we analyzed only patients with clinically localized disease, and thus, we couldn't compare those cases with locally advanced and metastatic disease between the two groups. Finally, due to the relative short follow-up period, we were not able to determine whether other important measures of outcome (metastases-free survival or overall survival) were different amongst the two groups. 
The present study is unique because it provides an insight concerning the epidemiology of PCa among Brazilian men in the extremes of socioeconomic conditions. As a developing country, Brazil has great socioeconomic disparities, and this is known to influence many factors that are related to cancer development and behavior, such as dietary habits, environmental exposures to risk factors, as well as access and utilization of health care services.

It's well known that improving diagnosis tests and surgical methods have extreme importance for public health management. However, only improving screening methods and training surgeons may not be sufficient to solve these problems if the government does not provide equal health care access to different socioeconomic layers of the population. In this way, the present study results could support the need to plan new strategies in PCa campaigns.

\section{CONCLUSIONS}

In a developing country, patients with $\mathrm{PCa}$ and a low SES were older, presented more aggressive disease characteristics and a high rate of biochemical recurrence. A low SES constituted an independent predictor for disease recurrence. Analysis of larger series with more variables must be performed to confirm theses results.

\section{CONFLICT OF INTEREST}

None declared.

\section{REFERENCES}

1. Jemal A, Siegel R, Ward E, Hao Y, Xu J, Thun MJ: Cancer statistics, 2009. CA Cancer J Clin. 2009; 59: 225-49.

2. Espey DK, Wu XC, Swan J, Wiggins C, Jim MA, Ward E, et al.: Annual report to the nation on the status of cancer, 1975-2004, featuring cancer in
American Indians and Alaska Natives. Cancer. 2007; 110: 2119-52.

3. Adler NE, Newman K: Socioeconomic disparities in health: pathways and policies. Health Aff (Millwood). 2002; 21: 60-76.

4. Cheng I, Witte JS, McClure LA, Shema SJ, Cockburn MG, John EM, et al.: Socioeconomic status and prostate cancer incidence and mortality rates among the diverse population of California. Cancer Causes Control. 2009; 20: 1431-40.

5. Clegg LX, Reichman ME, Miller BA, Hankey BF, Singh GK, Lin YD, et al.: Impact of socioeconomic status on cancer incidence and stage at diagnosis: selected findings from the surveillance, epidemiology, and end results: National Longitudinal Mortality Study. Cancer Causes Control. 2009; 20: 41735.

6. Miller DC, Litwin MS, Bergman J, Stepanian S, Connor SE, Kwan L, et al.: Prostate cancer severity among low income, uninsured men. J Urol. 2009; 181: 579-83; discussion 583-4.

7. Greene FL, Page DL, Fleming ID, et al.: AJCC Cancer Staging Manual, 6th edn. New York: SpringerVerlag, 2002; pp. 3-15.

8. Gleason DF: Histologic grading and clinical staging of prostatic carcinoma. In: Eble JN, Sauter G, Epstein JI, Sesterhenn IA (ed.), Pathology and Genetics of Tumours of the Urinary System and Male Genital Organs. Lyon, IARC Press. 2004: pp. 167214.

9. Brasil: Ministério do Planejamento, Orçamento e Gestão. Instituto Brasileiro de Geografia e Estatística [internet]. (cited april. 2009). Available from: http://www.ibge.gov.br.

10. Grossfeld GD, Chang JJ, Broering JM, Miller DP, Yu J, Flanders SC, et al.: Impact of positive surgical margins on prostate cancer recurrence and the use of secondary cancer treatment: data from the CaPSURE database. J Urol. 2000; 163: 1171-7.

11. Obek C, Sadek S, Lai S, Civantos F, Rubinowicz D, Soloway MS: Positive surgical margins with radical retropubic prostatectomy: anatomic site-specific pathologic analysis and impact on prognosis. Urology. 1999; 54: 682-8.

12. Kato RB, Srougi V, Salvadori FA, Ayres PP, Leite KM, Srougi M: Pretreatment tumor volume estimation based on total serum psa in patients with localized prostate cancer. Clinics (Sao Paulo). 2008; 63: 759-62. 
13. Godley PA, Schenck AP, Amamoo MA, Schoenbach VJ, Peacock S, Manning M, et al.: Racial differences in mortality among Medicare recipients after treatment for localized prostate cancer. J Natl Cancer Inst. 2003; 95: 1702-10.

14. Gilligan T: Social disparities and prostate cancer: mapping the gaps in our knowledge. Cancer Causes Control. 2005; 16: 45-53.
15. Tewari AK, Gold HT, Demers RY, Johnson CC, Yadav R, Wagner EH, et al.: Effect of socioeconomic factors on long-term mortality in men with clinically localized prostate cancer. Urology. 2009; 73: 624-30. Erratum in: Urology. 2009; 74: 239. Yood, Marianne Ulcickas [added].

\section{Correspondence address:}

Dr. Alberto A. Antunes

Rua Peixoto Gomide, 502/ 174-B

Sao Paulo, SP, 01409-000, Brazil

Fax: + $55113255-6372$

E-mail: antunesuro@uol.com.br 